\title{
Caracterização fenológica e exigência térmica de diferentes variedades de uvas viníferas em São Joaquim, Santa Catarina - Brasil
}

\author{
Phenological characterization and thermic requirement of distinct \\ grapevines varieties in São Joaquim, Santa Catarina-Brazil
}

\author{
Alberto Fontanella Brighenti ${ }^{\mathrm{I}}$ Emilio Brighenti ${ }^{\mathrm{II}}$ Valdir Bonin $^{\mathrm{II}}$ Leo Rufato ${ }^{\mathrm{III}}$
}

\section{RESUMO}

\begin{abstract}
A produção de uvas viníferas nas regiões de elevada altitude do estado de Santa Catarina é recente e há poucas informações disponíveis a respeito das características fenológicas e das exigências térmicas para as variedades utilizadas. O objetivo deste trabalho foi caracterizar o comportamento fenológico e determinar as exigências térmicas das variedades Chardonnay, Sauvignon Blanc, Cabernet Sauvignon, Merlot, Pinot Noir, Cabernet Franc e Sangiovese. A área experimental foi instalada na Estação Experimental da EPAGRI, localizada em São Joaquim (2817’39”'S; 4955'56”'W, altitude 1.415m). Os estádios fenológicos avaliados foram início da brotação, floração, mudança de cor das bagas e maturidade nos ciclos produtivos de 2004/05, 2005/06 e 2006/07. A exigência térmica das variedades foi calculada empregando-se o somatório de grausdia, considerando-se temperatura-base para a videira de $10^{\circ} \mathrm{C}$. Na colheita, a maturação tecnológica foi determinada através das análises de sólidos solúveis totais, acidez titulável e $\mathrm{pH}$. O ciclo das variedades viníferas avaliadas na região de São de Joaquim é mais longo do que o observado em outras regiões produtoras do Brasil. A duração térmica é um bom indicador de desenvolvimento das fases do ciclo da videira. Para as regiões com altitude acima de $1.300 \mathrm{~m}$, deve-se dar preferência para o plantio de variedades com ciclos entre 15 de setembro até 15 de abril.
\end{abstract}

Palavras-chave: regiões de altitude elevada, graus-dias, Vitis vinifera $L$.

\section{ABSTRACT}

The grapevine production in high altitude regions of southern Brazil is recent and there is little information about the phenological stages and thermal requirements of different grape varieties. The objective of this study was to determine the phenology and thermal requirements of Chardonnay, Sauvignon Blanc, Cabernet Sauvignon, Merlot, Pinot Noir, Cabernet Franc and Sangiovese. The experiment was conducted at the Experimental
Station of São Joaquim, EPAGRI, (28¹7'39” S; 4955'56” W, altitude 1,415 meters). The phenological stages evaluated were bud break, flowering, change in berry skin color and maturity, during seasons 2004/05, 2005/06 and 2006/07. Thermal requirements were calculated by degree-days, considering base temperature of $10^{\circ} \mathrm{C}$. At harvest, technological maturity was determined by analyses of total soluble solids, titratable acidity and $\mathrm{pH}$. It was observed a delay in vine cycle, because harvest occured later in this place than in other Brazilian regions. The thermal durations are a good indicator of vine development phases. Regions above $1,300 \mathrm{~m}$ should give preference to varieties with cycle between September 15 and April 15.

Key words: high altitude regions, degree-days, Vitis vinifera $L$.

\section{INTRODUÇÃO}

No estado de Santa Catarina, novas regiões produtoras de uvas viníferas estão surgindo em zonas de altitude acima de 1.000 metros. No entanto, o cultivo da videiranesses locaisémuito recente, comaproximadamente 10 anos, logo, é fundamental para o êxito dessa nova atividade econômica identificar variedades, adaptadas às condições dessas regiões, que sejam capazes de produzir uvas e consequentemente vinhos de alta qualidade. Uma das ferramentas mais importantes para identificar a adaptação de diferentes variedades em uma região, é a caracterização dos estádios fenológicos e da exigência térmica.

A caracterização das exigências térmicas da videira mediante o conceito de graus-dia é um

\footnotetext{
'Programa de Pós-graduação em Recursos Genéticos Vegetais (RGV), Universidade Federal de Santa Catarina (UFSC), Rodovia Admar Gonzaga, 1346, Itacorubi, 88040-900, Florianópolis, SC, Brasil. E-mail: brighenti_07@hotmail.com. Autor para correspondência.

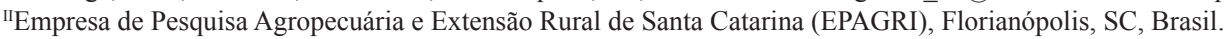

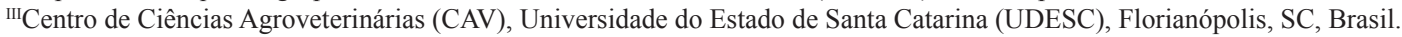


método eficiente para determinar, em diversas regiões, o tempo necessário entre o florescimento e a maturação dos frutos, ou qualquer fase fenológica, de diversas variedades (PEDRO JÚNIOR et al., 1994).

O conhecimento dos estádios fenológicos é uma exigência da viticultura moderna, uma vez que possibilita a racionalização e a otimização de práticas culturais, que são indispensáveis para o cultivo da videira. Por exemplo, a data da brotação possibilita a organização e racionalização da poda e a determinação da data do tratamento fitossanitário de inverno. A data da floração é fundamental para o monitoramento e controle das podridões do cacho e a data da maturação das uvas possibilita a organização dos trabalhos de campo (colheita e transporte) e da indústria (recebimento e uso de equipamentos enológicos) (MANDELLI et al., 2003).

A duração e a data de ocorrência dos diferentes estádios fenológicos da videira varia de acordo com a variedade, o clima e a localização geográfica do vinhedo (WEBB et al., 2007). A duração dos estádios fenológicos também está relacionada com a capacidade produtiva da planta, quando esses períodos são precoces e bem expressos, resultam em maiores produtividades (JONES \& DAVIS, 2000).

Este trabalho teve por objetivo caracterizar o desenvolvimento fenológico e determinar as exigências térmicas de sete variedades de uvas viníferas durante três ciclos consecutivos em regiões de altitude elevada de São Joaquim, Santa Catarina.

\section{MATERIAL E MÉTODOS}

O experimento foi realizado na Estação Experimental de São Joaquim - EPAGRI -, localizada em São Joaquim no estado de Santa Catarina, Brasil (2817'39'S, 4955'56"W, altitude de $1.415 \mathrm{~m})$. No vinhedo experimental, em que a coleção de variedades se localizava, foram selecionadas vinte plantas de cada variedade que foram acompanhadas por um período de três anos (2004/05, 2005/06 e 2006/07).

As variedades avaliadas foram

Chardonnay, Sauvignon Blanc, Pinot Noir, Cabernet Franc, Merlot, Cabernet Sauvignon e Sangiovese. Todas as variedades foram enxertadas sobre Paulsen 1103, sustentadas no sistema de espaldeira, podadas em cordão esporonado e plantadas no espaçamento de $3,00 \times 1,50 \mathrm{~m}$.

Os dados meteorológicos diários foram obtidos da estação agroclimática da Estação Experimental de São Joaquim, da EPAGRI. Os GrausDia (GD) de WINKLER (1980) foram calculados a partir do somatório das unidades térmicas entre o início da brotação e a colheita (maturidade das uvas), de acordo com a seguinte equação:

$\mathrm{Tm}>\mathrm{Tb}$; $\mathrm{GD}=\sum(\mathrm{Tm}-\mathrm{Tb})+[(\mathrm{TM}-\mathrm{Tm}) / 2], \quad$ quando $\mathrm{Tm} \leq \mathrm{Tb}$ e;

$\mathrm{GD}=\sum\left[(\mathrm{TM}-\mathrm{Tb})^{2} / 2 *(\mathrm{TM}-\mathrm{Tm})\right], \quad$ quando

$\mathrm{GD}=0$, quando $\mathrm{Tb} \geq \mathrm{TM}$;

Em que GD são os Graus-Dia; TM é a Temperatura máxima, em ${ }^{\circ} \mathrm{C}$; Tm é a Temperatura mínima, em ${ }^{\circ} \mathrm{C}$; $\mathrm{Tb}$ é a Temperatura-base de $10^{\circ} \mathrm{C}$.

A determinação da fenologia das plantas foi efetuada pela mesma pessoa, nos três ciclos estudados, através de observações visuais realizadas semanalmente após a poda. Oinício de brotação, a plena floração, a mudança de cor das bagas e a maturidade foram determinados segundo a classificação proposta por BAILLOD \& BAGGIOLINI (1993).

A data do início da brotação foi considerada quando $50 \%$ das gemas atingiram o estádio de ponta verde, quando começa a aparecer o jovem broto sobre as gemas. A data da plena floração foi considerada quando $50 \%$ das caliptras florais se separam da base do ovário.

A data da mudança de cor das bagas foi considerada quando $50 \%$ das bagas mudaram de coloração. Nesse caso, as bagas das variedades de película branca se tornam translúcidas e as bagas das variedades tintas adquirem uma coloração avermelhada. $\mathrm{O}$ período de maturidade foi considerado como a data da colheita e, para tal, foi considerada a maturação tecnológica das uvas.

As análises de maturação tecnológica foram realizadas no momento da colheita. Através do mosto, obtido com o esmagamento das bagas das uvas, foram determinados os Sólidos Solúveis Totais ( ${ }^{\circ}$ Brix), a Acidez Titulável (meq $\mathrm{L}^{-1}$ ) e o $\mathrm{pH}$ de cada variedade, conforme a metodologia proposta pelo OFFICE INTERNATIONAL DE LA VIGNE ET DU VIN (1990). Utilizou-se a estatística descritiva: média, desvio padrão e coeficiente de variação para a interpretação dos resultados.

\section{RESULTADOS E DISCUSSÃO}

Na tabela 1, encontram-se as datas dos principais estádios fenológicos das variedades estudadas. A variedade Chardonnay apresenta a brotação mais precoce, seguida pelas variedades Pinot Noir, Sangiovese e Cabernet Franc, Merlot, Sauvignon Blanc e Cabernet Sauvignon. Observou-se diferença média de 36 dias na data média de brotação da variedade mais precoce para a mais tardia. Situação semelhante ocorreu com MANDELLI et al. (2003) ao estudar o comportamento fenológico de diferentes variedades na Serra Gaúcha. 
Tabela 1 - Datas de ocorrência dos principais estádios fenológicos das variedades Chardonnay, Sauvignon Blanc, Cabernet Sauvignon, Merlot, Pinot Noir, Cabernet Franc e Sangiovese, em São Joaquim, SC, nos ciclos 2004/2005, 2005/2006 e 2006/2007.

\begin{tabular}{|c|c|c|c|c|c|c|c|c|}
\hline $\begin{array}{l}\text { Estádio } \\
\text { fenológico }\end{array}$ & Ciclo & Chardonnay & $\begin{array}{l}\text { Sauvignon } \\
\text { Blanc }\end{array}$ & $\begin{array}{l}\text { Cabernet } \\
\text { Sauvignon }\end{array}$ & Merlot & Pinot Noir & $\begin{array}{l}\text { Cabernet } \\
\text { Franc }\end{array}$ & Sangiovese \\
\hline \multirow{4}{*}{ Brotação } & $2004 / 05$ & $27 / 08$ & - & $24 / 09$ & $06 / 09$ & $31 / 08$ & $06 / 09$ & $04 / 09$ \\
\hline & $2005 / 06$ & $19 / 08$ & $30 / 09$ & $28 / 09$ & $16 / 09$ & $31 / 08$ & $02 / 09$ & $02 / 09$ \\
\hline & $2006 / 07$ & $08 / 08$ & $15 / 09$ & $20 / 09$ & $12 / 09$ & $17 / 08$ & 07/09 & $11 / 09$ \\
\hline & Média & $18 / 08 \pm 10$ & $22 / 09 \pm 11$ & $24 / 09 \pm 4$ & $11 / 09 \pm 5$ & $26 / 08 \pm 8$ & $05 / 09 \pm 3$ & $05 / 09 \pm 5$ \\
\hline \multirow{4}{*}{ Floração } & $2004 / 05$ & $04 / 11$ & - & $02 / 12$ & $29 / 11$ & $11 / 11$ & $23 / 11$ & $14 / 11$ \\
\hline & $2005 / 06$ & $22 / 11$ & $11 / 12$ & $15 / 12$ & $30 / 11$ & $23 / 11$ & $01 / 12$ & $30 / 11$ \\
\hline & $2006 / 07$ & $10 / 11$ & $22 / 11$ & $28 / 11$ & $22 / 11$ & $10 / 11$ & $22 / 11$ & $13 / 11$ \\
\hline & Média & $12 / 11 \pm 9$ & $01 / 12 \pm 13$ & $05 / 12 \pm 9$ & $27 / 11 \pm 4$ & $14 / 11 \pm 7$ & $25 / 11 \pm 5$ & $19 / 11 \pm 10$ \\
\hline \multirow{4}{*}{$\begin{array}{l}\text { Mudança } \\
\text { de Cor }\end{array}$} & $2004 / 05$ & $29 / 01$ & - & $09 / 02$ & $05 / 02$ & $14 / 01$ & $09 / 02$ & $26 / 01$ \\
\hline & $2005 / 06$ & $31 / 01$ & $10 / 02$ & $22 / 02$ & $06 / 02$ & $02 / 02$ & $15 / 02$ & $12 / 02$ \\
\hline & $2006 / 07$ & $25 / 01$ & $28 / 01$ & $31 / 01$ & $23 / 01$ & $08 / 01$ & $24 / 01$ & $20 / 01$ \\
\hline & Média & $28 / 01 \pm 3$ & $03 / 02 \pm 9$ & $10 / 02 \pm 11$ & $01 / 02 \pm 8$ & $18 / 01 \pm 13$ & $05 / 02 \pm 11$ & $29 / 01 \pm 12$ \\
\hline \multirow{4}{*}{ Maturidade } & $2004 / 05$ & $10 / 03$ & - & $02 / 05$ & $14 / 04$ & $10 / 03$ & $12 / 04$ & $25 / 03$ \\
\hline & $2005 / 06$ & $27 / 03$ & $27 / 03$ & $27 / 04$ & $19 / 04$ & $27 / 03$ & $05 / 04$ & $21 / 03$ \\
\hline & $2006 / 07$ & $03 / 03$ & $06 / 03$ & $19 / 04$ & $02 / 04$ & $06 / 03$ & $03 / 04$ & $19 / 03$ \\
\hline & Média & $13 / 03 \pm 12$ & $16 / 03 \pm 15$ & $26 / 04 \pm 7$ & $11 / 04 \pm 9$ & $14 / 03 \pm 11$ & $06 / 04 \pm 5$ & $21 / 03 \pm 3$ \\
\hline
\end{tabular}

As diferentes variedades possuem distintas datas de ocorrência para os principais estádios fenológicos. Essa diferença ocorre devido à variedade, clima e posição geográfica (JONES, 1997). Esse fato explica a relativa dificuldade em se estabelecer uma escala precisa de brotação para as principais variedades de uma região (JONES \& DAVIS, 2000).

Comparando-se a data média de início de brotação das variedades em São Joaquim (acima de 1.300 metros de altitude) com aquelas obtidas por MANDELLI et al. (2003) na Serra Gaúcha (em torno de 600 metros de altitude), constata-se que, em São Joaquim, a data de brotação da Cabernet Sauvignon e Merlot foi semelhante à registrada na Serra Gaúcha, entretanto, houve uma antecipação de 5 a 10 dias na brotação da Chardonnay, Pinot Noir e Cabernet Franc.

Acredita-se que as diferenças nas datas de brotação, entre os dois locais, estejam relacionadas com a necessidade de frio para a superação da dormência. Em locais mais frios, como São Joaquim, a brotação é antecipada porque as plantas atingem antes as horas de frio necessárias para a superação da dormência.

A classificação baseada na época de brotação é importante para os viticultores, pois permite que eles possam utilizar variedades de brotação precoce em locais com baixo risco de ocorrência de geadas tardias, e variedades de brotação tardia em locais propensos à esse fenômeno (MANDELLI et al., 2003). As variedades Chardonnay e Pinot Noir, com as suas datas de brotação no mês de agosto, estão especialmente expostas ao risco de danos por geadas, de ocorrência comum nas regiões de elevada altitude durante esse período.

As variedades com brotação mais precoce também apresentaram floração mais precoce. Observou-se uma diferença de aproximadamente 20 dias na data média de floração das variedades mais precoces, em relação à data média da floração das variedades mais tardias.

A variedade Chardonnay apresenta a data de colheita mais precoce, seguida pelas variedades Pinot Noir, Sauvignon Blanc, Sangiovese, Cabernet Franc, Merlot e Cabernet Sauvignon (Tabela 1).

Quando se compara as datas médias de colheita das variedades em São Joaquim com aquelas obtidas por MANDELLI et al. (2003) na Serra Gaúcha, constata-se o efeito da altitude elevada na duração do ciclo da videira. Em São Joaquim, houve um atraso de aproximadamente 45 dias na data da colheita das variedades Chardonnay, Pinot Noir e Cabernet Franc e um atraso de aproximadamente 60 dias na colheita de Merlot e Cabernet Sauvignon.

Em todos os anos avaliados, foi observado que a data de colheita da variedade Cabernet Sauvignon ocorreu após a segunda quinzena de abril. É importante destacar que essa variedade corre o risco de não completar sua maturação em anos 
particularmente frios e chuvosos, visto que, a partir da segunda quinzena de abril, registram-se quedas consideráveis na temperatura da região.

Os dados obtidos neste trabalho vão ao encontro do que foi dito por JONES (1997), o qual aponta que, em regiões de climas mais frios, com estação de crescimento mais curta, variedades de maturação precoce são mais indicadas, enquanto, em climas quentes, são recomendadas variedades de maturação tardia, já que, nessa situação, elas possuem condições de completar sua maturação.

Constatou-se que as variedades possuem pouca variabilidade na duração de cada subperíodo fenológico entre os anos avaliados (Tabela 2). As variedades estudadas nesse experimento são cultivadas em grande diversidade de climas e são reconhecidas como castas internacionais, talvez por isso sejam pouco sensíveis ao meio (LOPES et al., 2008). A variedade Sauvignon Blanc possui o ciclo mais curto e a variedade Sangiovese apresentou o ciclo mais longo (Tabela 2).

JONES \& DAVIS (2000) relataram que o tempo que transcorre entre um estádio e outro pode determinar se a zona agroclimática é adequada ou não para a variedade. Longos períodos podem determinar baixas produções e podem ser resultado de más condições climáticas ou que a variedade não está adaptada ao local em questão. Essa situação pode ser observada na duração cronológica dos períodos entre brotação e floração da variedade Chardonnay e entre a mudança de cor das bagas e a maturidade da Cabernet Sauvignon.

A maturação da uva engloba o período que inicia com a mudança de cor das bagas e termina na colheita. Dessa forma, outro critério utilizado para avaliar a adaptação de variedades em novas regiões de cultivo é a duração do subperíodo de mudança de cor das bagas até a maturidade das uvas. A duração média desse subperíodo foi de 41 dias para Sauvignon Blanc, 45 para Chardonnay, 55 para Pinot Noir, 60 para Cabernet Franc, 70 para Merlot, 75 para Cabernet Sauvignon e 82 dias para Sangiovese. FREGONI (1998) argumenta que variedades que possuem esse período mais curto podem se adaptar mais facilmente a climas mais frios e altitudes mais elevadas.

Ainda na tabela 2, observam-se as durações térmicas (DT) e os coeficientes de variação

Tabela 2 - Duração cronológica média (DC, dias), duração térmica média (DT, Graus-dia) e coeficiente de variação (CV, \%) das fases do ciclo vegetativo, brotação - floração (Brot. - Flor.), floração - mudança de cor das bagas (Flor. - Mud. Cor), mudança de cor das bagas - maturidade (Mud. Cor - Mat.), brotação - mudança de cor das bagas (Brot. - Mud. Cor) e brotação - maturidade (Brot. Mat.), das variedades Chardonnay, Sauvignon Blanc, Cabernet Sauvignon, Merlot, Pinot Noir, Cabernet Franc e Sangiovese, em São Joaquim, SC, nos ciclos 2004/2005, 2005/2006 e 2006/2007.

\begin{tabular}{|c|c|c|c|c|c|c|c|c|c|c|}
\hline & \multicolumn{2}{|c|}{----Brot. - Flor.---- } & \multicolumn{2}{|c|}{--Flor. - Mud. Cor-- } & \multicolumn{2}{|c|}{--Mud. Cor - Mat.-- } & \multicolumn{2}{|c|}{--Brot. - Mud. Cor-- } & \multicolumn{2}{|c|}{----Brot. - Mat. ---- } \\
\hline & $\mathrm{DC}$ & $\mathrm{CV}$ & $\mathrm{DC}$ & $\mathrm{CV}$ & $\mathrm{DC}$ & $\mathrm{CV}$ & $\mathrm{DC}$ & $\mathrm{CV}$ & $\mathrm{DC}$ & $\mathrm{CV}$ \\
\hline Chardonnay & 86 & $15,1 \%$ & 77 & $10,5 \%$ & 45 & $19,3 \%$ & 163 & $4,7 \%$ & 208 & $6,0 \%$ \\
\hline Sauvignon Blanc & 70 & $4,0 \%$ & 64 & $6,6 \%$ & 41 & $13,8 \%$ & 134 & $1,1 \%$ & 175 & $2,4 \%$ \\
\hline Cabernet Sauvignon & 72 & $7,2 \%$ & 67 & $4,3 \%$ & 75 & $9,6 \%$ & 139 & $7,1 \%$ & 214 & $4,9 \%$ \\
\hline Merlot & 77 & $8,7 \%$ & 66 & $5,3 \%$ & 70 & $3,0 \%$ & 143 & $6,7 \%$ & 212 & $4,4 \%$ \\
\hline Pinot Noir & 80 & $9,0 \%$ & 65 & $9,2 \%$ & 55 & $3,6 \%$ & 145 & $6,2 \%$ & 200 & $4,0 \%$ \\
\hline Cabernet Franc & 81 & $9,8 \%$ & 73 & $10,7 \%$ & 60 & $16,9 \%$ & 154 & $9,2 \%$ & 214 & $2,5 \%$ \\
\hline Sangiovese & 74 & $15,9 \%$ & 72 & $4,5 \%$ & 82 & $14,8 \%$ & 146 & $11,0 \%$ & 228 & $3,1 \%$ \\
\hline \multirow[t]{3}{*}{ Média } & 77 & $9,9 \%$ & 69 & $7,3 \%$ & 68 & $11,6 \%$ & 145 & $6,6 \%$ & 214 & $3,9 \%$ \\
\hline & \multicolumn{2}{|c|}{----Brot. - Flor. ---- } & \multicolumn{2}{|c|}{--Flor. - Mud. Cor-- } & \multicolumn{2}{|c|}{--Mud. Cor - Mat.-- } & \multicolumn{2}{|c|}{--Brot. - Mud. Cor-- } & \multicolumn{2}{|c|}{----Brot. - Mat. ---- } \\
\hline & DT & $\mathrm{CV}$ & DT & CV & DT & $\mathrm{CV}$ & DT & $\mathrm{CV}$ & DT & CV \\
\hline Chardonnay & 347 & $10,3 \%$ & 482 & $1,2 \%$ & 467 & $5,8 \%$ & 829 & $8,7 \%$ & 1.296 & $3,9 \%$ \\
\hline Sauvignon Blanc & 348 & $8,8 \%$ & 535 & $6,2 \%$ & 289 & $6,1 \%$ & 905 & $7,1 \%$ & 1.194 & $3,5 \%$ \\
\hline Cabernet Sauvignon & 387 & $10,3 \%$ & 521 & $3,9 \%$ & 522 & $18,9 \%$ & 908 & $2,3 \%$ & 1.430 & $6,8 \%$ \\
\hline Merlot & 344 & $4,3 \%$ & 516 & $2,2 \%$ & 541 & $9,5 \%$ & 861 & $1,4 \%$ & 1.402 & $3,2 \%$ \\
\hline Pinot Noir & 329 & $8,6 \%$ & 472 & $14,0 \%$ & 434 & $6,0 \%$ & 801 & $11,0 \%$ & 1.235 & $4,3 \%$ \\
\hline Cabernet Franc & 355 & $9,5 \%$ & 565 & $12,2 \%$ & 383 & $12,7 \%$ & 920 & $10,5 \%$ & 1.303 & $7,9 \%$ \\
\hline Sangiovese & 318 & $12,5 \%$ & 541 & $8,3 \%$ & 452 & $9,6 \%$ & 859 & $12,7 \%$ & 1.311 & $5,0 \%$ \\
\hline Média & 347 & $9,2 \%$ & 519 & $6,9 \%$ & 441 & $9,8 \%$ & 869 & $6,1 \%$ & 1.310 & $4,9 \%$ \\
\hline
\end{tabular}


das fases do ciclo de desenvolvimento de cada variedade. Houve uma redução nos coeficientes de variação das durações térmicas em comparação com os das durações cronológicas. Segundo LOPES et al. (2008), esse fato ocorre porque as durações térmicas possuem a vantagem de minimizar ou eliminar o efeito das variações interanuais da temperatura no desenvolvimento das plantas. O coeficiente de variação da duração térmica do ciclo completo é semelhante ao da duração cronológica.

A fase "mudança de cor das bagas maturidade" apresentou a maior variabilidade, assim como o relatado por LOPES et al. (2008), que atribuem esse fato às dificuldades práticas na observação dessas fases, quando comparadas com a brotação e a floração.

As variedades Cabernet Sauvignon e Merlot apresentaram o maior requerimento térmico em seus ciclos, com 1.430 e 1.402 graus-dia, respectivamente (Tabela 2). A precocidade das variedades Pinot Noir e Chardonnay também foi observada, provavelmente porque elas necessitam de menos graus-dia no subperíodo de maturação (Tabela 2).

Em consequência do regime térmico, a duração do período que vai da brotação à maturidade das uvas é maior em São Joaquim e nas regiões de altitude elevada. Para a variedade Cabernet Sauvignon, a duração média do ciclo é de 214 dias e a duração térmica média para o período avaliado foi 1.430 graus-dias.

As diferenças na duração do ciclo também são observadas em outras partes do Brasil e do mundo. Por exemplo, na Serra Gaúcha, o período de brotação à maturidade da Cabernet Sauvignon é de 152 dias e a exigência térmica é de 1.543 grausdia (BRIGHENTI \& TONIETTO, 2004), em clima subtropical, no Paraná, de 130 dias (SATO, et al., 2011), em Bordeaux, na França, é de 193 dias (JONES \& DAVIS, 2000). Já na Patagônia, a duração do ciclo se assemelha às regiões de altitude de Santa Catarina, com 213 dias (LLORENTE, 2007).

Para a variedade Merlot, o período da brotação à maturidade, em São Joaquim, dura em média 212 dias e a duração térmica média para o período avaliado foi 1.402 graus-dia, enquanto que, na Serra Gaúcha, esse período é 148 dias e a duração térmica é de 1.385 graus-dia (BRIGHENTI \& TONIETTO, 2004).

As variedades Merlot e Cabernet Franc apresentaram os maiores teores médios de sólidos solúveis totais. No entanto, é importante enfatizar que, nos três ciclos avaliados, todas as variedades estudadas atingiram, na colheita, níveis de sólidos solúveis totais apropriados para a elaboração de vinhos de qualidade $\left(19,0\right.$ a $22,0^{\circ}$ Brix) (Tabela 3$)$. Resultados semelhantes foram obtidos por GRIS et al. (2010), quando estudaram as variedades Cabernet Franc, Merlot e Sangiovese na mesma região durante os ciclos 2005/2006 e 2006/2007.

Os valores obtidos de $\mathrm{pH}$ variaram de 3,13 a 3,53 nas variedades avaliadas (Tabela 3). De acordo com RIZZON \& MIELE (2003), esses valores de $\mathrm{pH}$ são adequados para elaboração de vinhos de qualidade.

Os valores de acidez titulável obtidos nos três ciclos avaliados também são adequados para elaboração de vinhos (Tabela 3). GRIS et al. (2010) e BORGHEZAN et al. (2011) também encontraram valores similares quando estudaram as variedades Cabernet Franc, Merlot, Sangiovese, Cabernet Sauvignon e Sauvignon Blanc na região de São Joaquim, SC. Destaca-se que, graças ao clima frio das regiões de altitude elevada, a degradação dos ácidos será sempre mais lenta e, como consequência, os teores de acidez titulável sempre serão mais elevados nas uvas e vinhos produzidos nesse local.

O período de ocorrência dos estádios fenológicos está relacionado com a capacidade da

Tabela 3 - Sólidos solúveis totais, acidez total titulável e pH das variedades Chardonnay, Sauvignon Blanc, Cabernet Sauvignon, Merlot, Pinot Noir, Cabernet Franc e Sangiovese, em São Joaquim, SC, nos ciclos 2004/2005, 2005/2006 e 2006/2007.

\begin{tabular}{|c|c|c|c|c|c|c|}
\hline \multirow[b]{2}{*}{ Variedade } & \multicolumn{2}{|c|}{---Sólidos solúveis totais $\left({ }^{\circ} \mathrm{Brix}\right)$-- } & \multicolumn{2}{|c|}{----Acidez titulável (meq L L-1) ---- } & \multicolumn{2}{|c|}{------------pH- } \\
\hline & Média & $\mathrm{CV}$ & Média & $\mathrm{CV}$ & Média & $\mathrm{CV}$ \\
\hline Chardonnay & 19,2 & $9,4 \%$ & 128,33 & $11,5 \%$ & 3,22 & $2,4 \%$ \\
\hline Sauvignon Blanc & 20,2 & $14 \%$ & 147,75 & $24,0 \%$ & 3,15 & $2,2 \%$ \\
\hline Cabernet Sauvignon & 20,9 & $1,5 \%$ & 122,67 & $9,27 \%$ & 3,37 & $4,5 \%$ \\
\hline Merlot & 21,7 & $6,9 \%$ & 113,33 & $15,5 \%$ & 3,43 & $6,7 \%$ \\
\hline Pinot Noir & 19,6 & $2,7 \%$ & 135,00 & $12,3 \%$ & 3,33 & $7,1 \%$ \\
\hline Cabernet Franc & 21,7 & $1,1 \%$ & 113,67 & $8,7 \%$ & 3,53 & $7,1 \%$ \\
\hline Sangiovese & 19,1 & $5,3 \%$ & 127,33 & $5,1 \%$ & 3,13 & $3,7 \%$ \\
\hline
\end{tabular}


videira em produzir frutos. Estádios fenológicos precoces e bem definidos normalmente resultam em maiores produtividades. Além disso, o momento em que ocorrem os estádios fenológicos pode ser relacionado com a qualidade das uvas produzidas (JONES, 1997).

\section{CONCLUSÃO}

O ciclo das variedades viníferas avaliadas na região de São de Joaquim é mais longo do que o observado em outras regiões produtoras do Brasil. Para as regiões com altitude acima de $1.300 \mathrm{~m}$, devese dar preferência a variedades que possuem ciclo intermediário, com duração entre 15 de setembro até 15 de abril. Nos três ciclos avaliados, as variedades estudadas produziram uvas com características adequadas para a produção de vinhos finos de qualidade.

\section{REFERÊNCIAS}

BAILLOD, M.; BAGGIOLINI, M. Les stades répères de la vigne. Revue Suisse de Viticulture Arboriculture Horticulture, Lausanne, v.28, p.7-9, 1993.

BORGHEZAN, M. et al. Comportamento vegetativo e produtivo da videira e composição da uva em São Joaquim, Santa Catarina. Pesquisa Agropecuária Brasileira, Brasília, v.46, p.398-405, 2011.

BRIGHENTI, E.; TONIETTO, J. O clima de São Joaquim para a viticultura de vinhos finos: classificação pelo sistema CCM geovitícola. In: CONGRESSO BRASILEIRO DE FRUTICULTURA, 8., 2004, Florianópolis, SC. Anais eletrônicos... Florianópolis: SBF, 2004. 4p. (CD-ROM).

FREGONI, M. Viticoltura di qualitá. Verona: Edizione I'Informatore Agrário, 1998. 707p.

GRIS, E.F. et al. Phenology and ripening of Vitis vinifera (L.) grape varieties in São Joaquim, southern Brazil: a new South
American wine growing region. Ciencia e Investigación Agraria, Santiago, v.37, p.61-75, 2010.

JONES, G.V. A synoptic climatological assessment of viticultural phenology. 1997. 394f. Ph.D. Dissertation, University of Virginia, Department of Environmental Sciences.

JONES, G.; DAVIS, R. Climate influences on grapevine phenology, grape composition, and wine production and quality for Bordeaux, France. American Journal of Enology and Viticulture, Davis, v.51, p.249-261, 2000.

LLORENTE, A. Fenologia y madurez de uva para vinificar. Argentina: Instituto Nacional de Tecnología Agropecuaria (INTA), 2007. Acesso em: 12 jun. 2012. Online. Disponível em: $<\mathrm{http}: / /$ www.inta.gov.ar/ALTOVALLE/info/biblo/rompecabezas/pdfs/ rompe39_llorente.pdf>.

LOPES, J. et al. Exigências térmicas, duração e precocidade de estados fenológicos de castas da colecção ampelográfica nacional. Ciência e Técnica Vitivinícola, Dois Portos, v.23, p.61-71, 2008.

MANDELLI, F. et al. Fenologia da videira na Serra Gaúcha. Pesquisa Agropecuária Gaúcha, Porto Alegre, v.9, p.129-144, 2003.

OFFICE INTERNATIONAL DE LA VIGNE ET DU VIN. Recueil des méthodes internationales d'analyse des vins et des moûts. Paris, 1990. 368p.

PEDRO JÚNIOR, M.J. et al. Previsão agrometeorológica da data de colheita para a videira 'Niágara Rosada'. Bragantia, Campinas, v.53, p.113-119, 1994.

IZZON, L.A.; MIELE, A. Avaliação da cv. 'Merlot' para elaboração de vinho tinto. Ciência e Tecnologia de Alimentos, Campinas, v.23, p.156-161, 2003.

SATO, A.J. et al. Fenologia, produção e composição do mosto da 'Cabernet Sauvignon' e 'Tannat' em clima subtropical. Revista Brasileira de Fruticultura, Jaboticabal, v.33, p.491-499, 2011.

WEBB, L.B. et al. Modelled impact of future climate change on the phenology of winegrapes in Australia. Australian Journal of Grape and Wine Research, v.13, p.165-175, 2007.

WINKLER, A.J. Viticultura. 6.ed. México: Editorial Continental, 1980. 791p. 